\title{
An Automatic Fringe Counting Interferometer for use in the Calibration of Line Scales
}

\author{
Herbert D. Cook and Louis A. Marzetta
}

(January 31, 1961)

\begin{abstract}
A reversible fringe counting interferometer is described in which mechanical, optical, and electronic adjustments are maintained stable by servomechanism control or by balancing. Mirror parallelism is achieved by detecting the angular error electronically and correcting by means of barium titanate actuators. An electronic interpolator permits recording of the court in digital form to 0.01 fringe without ambiguity. A rate of more than 1,200 fringes per second has been achieved over a range of 14 centimeters. Higher counting rates are possible over shorter ranges. Design factors and details are discussed. A correction factor is derived for the error introduced by finite collimation of the interferometer beam.
\end{abstract}

\section{Introduction}

As part of a program to take advantage of the new radiation standard of length, an experimental reversible fringe counting interferometer was developed for use in the calibration of line scales at NBS. At the present time, line scales are calibrated by comparison with a material standard, Meter bar No. 27, the length of which was derived from the International Prototype Meter maintained by the Bureau International des Poids et Mesures at Sevres in France.

Calibration with respect to the wavelength of a suitable radiation such as the lines of $\mathrm{Kr} 86$ or $\mathrm{Hg}$ 198 has several advantages over the use of a material standard. The wavelength standard is stable, easily reproducible in any laboratory, and can be accurately compared with the international standard. By means of fringe counting and interpolating techniques, any arbitrary length within the range of interference can be determined with great precision.

Since specially trained personnel are required for calibrating line scales and the work is tedious, the number of lines that can be observed in a working day is limited. As a result, some scales with many graduations can only be partially calibrated. The use of a mechanized instrument incorporating an interferometer and a photoelectric microscope for detecting the position of the lines of the scales is expected to be of help in making more complete calibrations.

In the use of an instrument, the operation of which requires many exacting procedures, the accuracy of measurement depends to some extent on the skill of the operator. Mechanization of such an instrument reduces the dependence on the operator and in some cases permits remotely controlled operation, allowing closer control of the environmental conditions. Carrying the mechanization one step further, automatic control is important if full advantage is to be taken of mechanization of the instrument, and the operator freed from full time attention to the details of the calibration process.

\subsection{Problems Encountered in the Design of an Interferometer}

The principles of operation of fringe counting interferometers have been extensively described in the literature. ${ }^{1}$ However, problems still exist in the application of the principles to a practical instrument.

The basic problem in measuring length with an interferometer is to determine the fringe order accurately as the position of one mirror changes with respect to the other. The number of fringes counted between two positions of the moving mirror is the number of half wavelengths of light in the displacement. If the displacement corresponds to a length to be measured, the value of the integral and fractional fringe count then represents a measurement of the length in half wavelengths. The fringes must be detected and analyzed with sufficient accuracy to allow inference to be made of the lengths represented by them. Any factors that influence the accuracy of measurement must be either under control during operation or be measured and record ed at the time.

Among the more serious problems is that of the mechanical precision required in the mechanism for moving the mirror. In some applications, corner reflectors have been used to eliminate the effect of angular rotation of the moving carriage, but in this application it was considered that their use did not constitute a suitable solution of the problem. If the carriage rotates in an unknown manner, the relationship between the center of reflection and the item being measured is in doubt. It is believed that more confidence can be placed in the accuracy of calibrations if plane mirrors are used and their parallelism maintained by electronic and mechanical means.

Methods must also be provided for overcoming errors which may arise with variations in intensity of the light source and for maintaining necessary adjustments correct throughout the period required for a calibration.

1 See bibliography at the end of this paper. 
The method of interpolation must be applicable to operation without manipulation by an operator and yet maintain a precision to the specified tolerance. A sine-cosine method of interpolation requires that the amplitudes and phase angles of the quadrature fringe signals be held always within close limits.

Solutions to these problems have been incorporated in an experimental instrument to be described in this paper. This was built to prove the design for a larger full scale instrument to be used in the length calibration laboratory.

\section{Optical Considerations}

\subsection{Interferometer}

A diagram of the optical arrangement is shown in figure 1. The Michelson type of interferometer is especially suited to the fringe counting application because the fringe intensity varies sinusoidally with mirror separation. Also, the range of travel of the moving mirror extends continuously on both sides of the zero-order fringe, allowing a total range double the maximum mirror separation at which fringes can be obtained.

Circular fringes are used in order to obtain the maximum possible light flux through the interferometer and to establish a mechanical symmetry in the system. Efficient use of light is important because of the low source intensity and the small apertures required at large mirror separations, the intensity being a limiting factor on the counting distance and the counting rate.

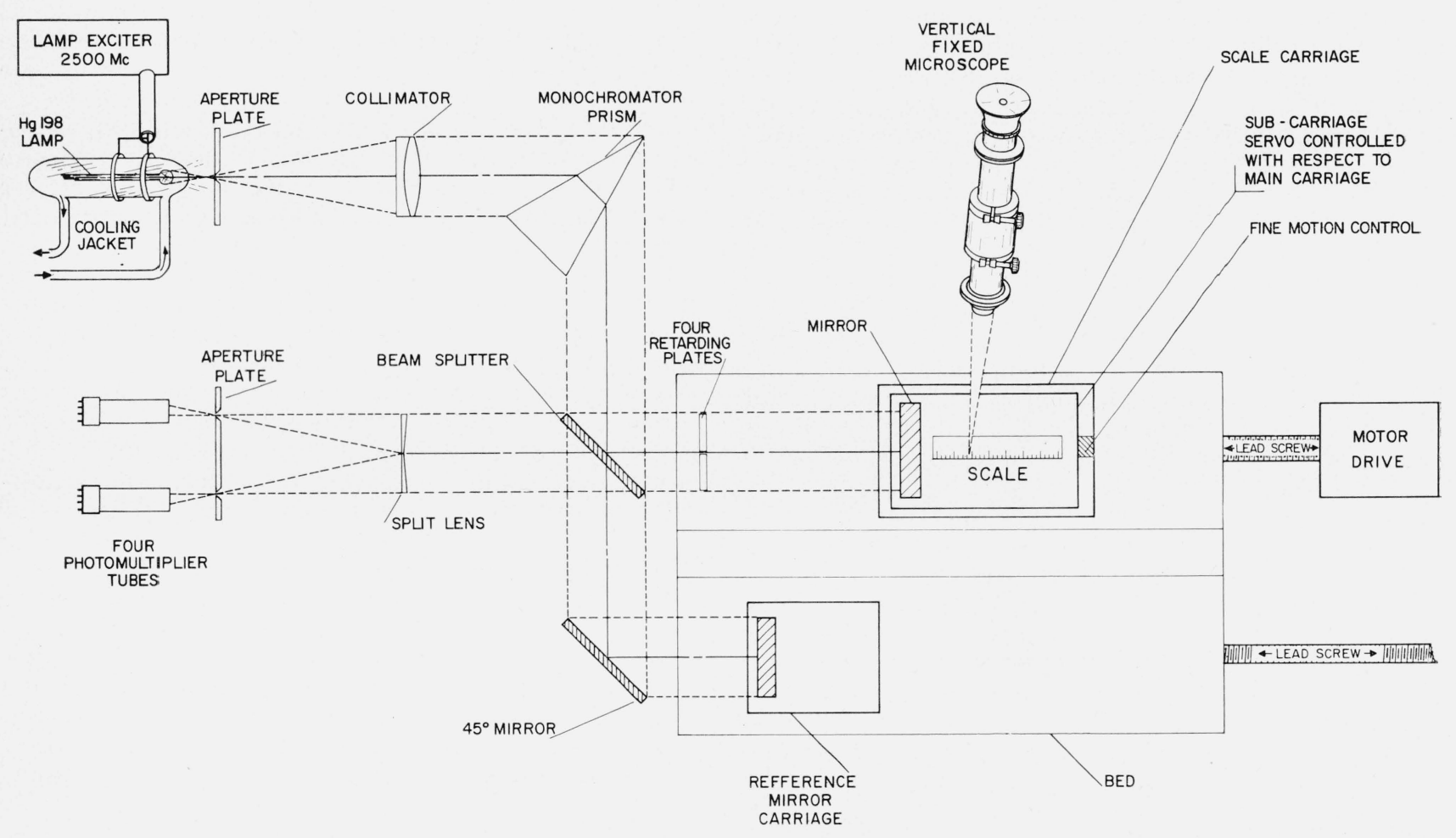

Figure 1. Layout of interferometor.
Two aperture plates are shown; the first at the light source determines the maximum angle, $\theta_{\max }$, at which light may pass through the interferometer. The second, placed in the image plane, has larger openings and is used to limit any stray light that might reach the phototubes and also to act in conjunction with a dispersing prism to form a monochromator.

The limit $\left(\theta_{\max }\right)$ of the solid angle of rays passing through the aperture and the interferometer depends on the maximum mirror separation at which reliable counting is required. At greater separations, more than one order of interference occurs in the light reaching the phototube, and the fringe contrast is seriously reduced. The radius of the maximum allowable aperture may be taken as approximately

$$
r \cong f \theta \cong f \sqrt{\frac{\lambda}{D_{\max }}}
$$

where $D_{\max }$ is twice the maximum mirror separation, lens, and $\lambda$ is the wavelength of the light. This relationship is derived in appendix 2 in addition to a correction factor for an error in the measurement of length due to the use of a finite aperture. Accurate centering of the aperture is also required.

The beam is divided into four parts, each quadrant producing a separate set of fringes which are detected by a separate phototube. This divides the interferometer into essentially four separate interferometers using portions of the same mirrors. The signals from the four phototubes are combined in $f$ is the focal length of the collimating or objective 
different ways for use in parallelism control, stabilization, counting, and interpolation.

In order to take advantage of the 4-phototube arrangement and to obtain the sine and cosine voltages necessary for reversible counting and interpolation, the optical path difference in each quadrant is adjusted so that a displacement of onequarter fringe exists between adjacent quadrants. Beginning with one quadrant as a reference, the fringe phases are respectively, in rotation, $0^{\circ}, 90^{\circ}$, $180^{\circ}$, and $270^{\circ}$.

The adjustment of phase is made by means of a retarding plate placed in each quadrant, the effective path length varying with the angle of tilt of the plate. Very close adjustment of the relative phase of the fringes can be made in this manner.

In the form of Michelson interferometer used, the fringe contrast is always less than unity at zero order because of an extra reflecting surface in one beam. The contrast is decreased in proportion to the ratio of the intensities of the two beams and this depends on the square of the coefficient of reflection of the 45-degree mirror. For this reason, the efficiency of this mirror is more important than that of any other single part of the optical system. A coefficient of 0.80 for an aluminized 45-degree mirror gives a maximum con trast of 0.64 .

The effect of less-than-unity-fringe contrast on the fringe signal output of the phototubes is to decrease the signal-to-noise ratio by approximately the same factor as the contrast. A dielectric coating on the 45-degree mirror with a coefficient greater than 95 percent is possible and would make the effect negligible.

Although the intensity of one beam is reduced by an extra reflection, that of the other is also reduced by the retarding plates, tending to balance the beam intensities and to increase the contrast and signal to noise ratio slightly.

\subsection{Light Source}

A suitable light source for measurement by interferometry is one which has high intensity and a spectral line width as narrow as possible, since the length over which interference fringes can be obtained (the coherence length) varies in reciprocal relationship to the width of the spectral line. The light sources available today have a coherence length of somewhat less than a half meter, but improved sources are expected to be available in the near future.

Although the orange line of $\operatorname{Kr} 86$ is the primary standard of length, the Meggers $\mathrm{Hg} 198$ lamp is probably a more suitable source for high-speed fringe counting since the intensity of the lines is higher. Also it is more convenient to operate.

The $\mathrm{Hg} 198$ electrodeless discharge lamp is excited by a high-frequency generator at a frequency which should be higher than $200 \mathrm{Mc} / \mathrm{s}$. A microwave source operating at $2,500 \mathrm{Mc} / \mathrm{s}$ has been convenient. The lamp is used end-on and is cooled in order to reduce Doppler broadening and self-reversal of the lines. Immersion in flowing water is a simple method of cooling, but absorption of the exciting energy makes coupling from the source inefficient. Immersion in a jacket with recirculated, cooled oil has been quite satisfactory. For experimental work, selection of the green or blue lines was made by means of pairs of Wratten filters, 58 and $77 \mathrm{~A}$ for the green, and $47 \mathrm{~B}$ and $34 \mathrm{~A}$ for the blue, but considerably more light intensity can be obtained if a prism monochromator is used.

\subsection{Phototubes}

In order to make use of the interference fringes generated in the interferometer, it is necessary to convert the light flux into an equivalent electrical signal by means of a phototube. Since phototubes are available with operating characteristics covering a wide range, a few words might be said about the factors involved in the selection of a tube for the application.

There are two principal sources of noise within the phototube, noise associated with the presence of light at the photocathode and noise generated by the socalled dark current. A typical photomultiplier tube, in total darkness, can have a dark current flow due to thermionic and other types of electron emission that is equivalent to the current that would result from a radiant flux of about $8 \times 10^{-10}$ lumens when operated at room temperature. Measurement with the Michelson interferometer used in the described equipment show that the light flux at the phototubes is approximately $1.5 \times 10^{-8}$ lumens when an aperture is selected for a measurement range of about $15 \mathrm{~cm}$. Since the dark current is at least one order of magnitude below the current due to this value of light flux, it can be neglected, as the signal noise $\left(N_{S}\right)$ and the dark current noise $\left(N_{D}\right)$ add as $\sqrt{N_{S}{ }^{2}+N_{D}{ }^{2}}$. If the light source were less intense, or the aperture reduced in order to obtain a longer maximum interference path length, the dark current would become important.

The noise associated with the presence of light flux is similar to shot noise in an electron tube and is due to the finite number of electrons per unit time in the current. Although the average phototube current is constant for a given incident light flux, the instantaneous current has a random variation about the average due to the noise. It can be shown that this noise current varies as the square root of the electron current ${ }^{2}{ }^{2}$ whereas the electron current varies directly with the light flux. Therefore the signal-to-noise ratio is proportional to the square root of the light flux.

The magnitude of the noise that can be tolerated in the fringe signal must be viewed from a consideration of the statistical behavior of the instantaneous noise pulses. In the length of one meter, the number of fringes to be counted is in the vicinity of $4,000,000$, depending on the wavelength. If the noise component amplitude is assumed to have a normal distribution, a calculation will show that the signal-tonoise ratio must be greater than $6: 1$ if the probability

\footnotetext{
2 Schottky's theorem, W. Schottky, Ann Physik Vol. 57, 541, (1918).
} 
of a noise pulse exceeding the signal in amplitude is to be less than one in $5 \times 10^{8}$ fringes when the counter is operating at the maximum rate. The input circuit used with the counter described later reduces the probability of miscount under these conditions.

The quantum efficiency of a photocathode is the ratio of the number of electrons released to the number of photons incident on its surface. The efficiency of selected S11 type cathode materials may be of the order of 10 to 15 percent. Recently a multi-alkali photocathode has been made available with an efficiency near 20 percent at $4200 \mathrm{~A}$. Furthermore, this new material offers a broader response curve over the visual spectrum plus an improvement in efficiency of 3 to 6 times over conventional photocathodes in the red region. Since the orange line of krypton lies in this region, this tube will prove valuable for use with the standard light source.

Measurements with the present equipment have indicated a potential due to the light flux of about $1 \mathrm{v}$ root mean square at the photomultiplier anode. Since a level of about $5 \mathrm{v}$ is needed at the trigger circuits of the counters, it may be necessary to provide a $d-c$ amplifier at the output of each phototube. The 10-stage multiplier tube used in the experimental equipment has a current amplification of about 600 ,000. A 14-stage photomultiplier operating at a higher supply voltage would have an amplification approaching $9 \times 10^{6}$, eliminating the need for a d-c amplifier, but at a considerable increase in the tube cost.

A small shift in the $\mathrm{d}-\mathrm{c}$ level at the phototube output due to the fatigue effect can occur under some conditions, with no change of light level. ${ }^{2 a}$ This can be reduced by operating the tube at reduced anode current. In the described system, the phototube anode load resistor is of the order of 5 megohms, and with the assigned voltage source, the anode current is less than $10 \mu \mathrm{a}$. An attempt to further lower the current by increasing the load resistor would result in a reduction of the response bandwidth. It was found to be essential to protect the phototubes from exposure to light levels that are in excess of their normal operating incidence flux. Fatigue effects of this sort usually persist for minutes or hours depending on the level of exposure. Because of the low level of flux present in the fringe pattern, the difference from normal operation to complete darkness that prevails with the equipment off does not result in an appreciable fatigue effect.

\section{Electrical Circuits}

A block diagram of the electronic circuits is shown in figure 2. The functions of the circuits include combining the outputs of the phototubes to produce stabilized sine and cosine signals, counting, interpolating, and digital readout of the fringe count data. Also, the information necessary for correction of parallelism is derived and applied to actuators which maintain the moving carriage always in correct orientation. Remote controls and monitoring of operations are also required of the electronic system.

2a See DuMont in bibliography.



Figure 2. Block diagram of electronic circuits. 


\subsection{Stabilization of the Electrical Fringe Signal}

The fringes in the interferometer are sensed as fluctuations in the light flux reaching the phototubes. The flux has the form of a fixed value around which the intensity varies as a sine function of the optical path difference. The amplitude of variation depends on optical factors which determine the fringe contrast, such as the flatness of the optical surfaces, reflection ratio of the beam splitter, the optical path difference and the spectral line width of the source. Since the fringe information lies in the variation only, it is necessary to isolate the signal in the form of a sinusoidal function with a baseline of zero. This is done conveniently by making use of the 4-phototube arrangement.

By choice of the effective thickness of the four plates in the interferometer beam, the phase retardation is adjusted so that there is an advance of onequarter fringe between adjacent quadrants in rotation. Diagonally opposite quadrants are thus in phase opposition. Taking the electrical difference between diagonal phototubes results in a signal which has twice the amplitude of each and a baseline of zero. The baseline remains zero with variations in the intensity of the light source, although the amplitude of the resulting sinusoidal voltage is proportional to the intensity. A further discussion is given in appendix 1. Two outputs are available, one from each pair of diagonal phototubes, one representing the sine function of distance and the other the cosine. These are used to operate the bidirectional counter and the interpolator. The signal-to-noise ratio in the output from each of the diagonal pairs is $\sqrt{2}$ times that for each phototube since the signals are added coherently and the noise noncoherently. Precise adjustments of phasing can be made easily to within $1 / 400$ th fringe. The accuracy of the sine and cosine voltages thus obtained is such that interpolation to better than $1 / 100$ th fringe is practical by means of sine-cosine methods.

Circuit Details. As shown in figure 3, the fringe signals generated at the four phototubes are directed to individual amplifiers via cathode followers. The combination of anode resistance and circuit capacitance establishes a bandwidth of about $1,200 \mathrm{c} / \mathrm{s}$. The sums and differences are taken for the diagonal pairs $A C$ and $B D . A-C$ represents the sine fringe signal, and $\mathrm{B}-\mathrm{D}$ represents the cosine fringe signal. The sums $\mathrm{A}+\mathrm{C}$ and $\mathrm{B}+\mathrm{D}$ are used for gain level adjustment.

To provide for balancing and adjustment of the photomultiplier gains, the sums and differences of each pair of diagonal phototube outputs are indicated on zero-center meters. The four meters have, associated with each, a variable resistor whose operation influences the supply voltage of the photomultiplier tubes in a manner such that its related meter is nulled. This is done while the interferometer is in operation.

\subsection{Reversible Counter}

The fringes sensed by the phototubes between two positions of the moving interferometer mirror are counted by an electronic counter and the total indicated as an integral number.

The counter must be reversible in order to insure that the fringe count is true at the end of a measurement, at which point the motion of the interferometer may be reversed to correct an overshoot. This may occur, since in the intended application it is convenient if the interferometer is able to stop at each measurement. Other requirements of a suit-

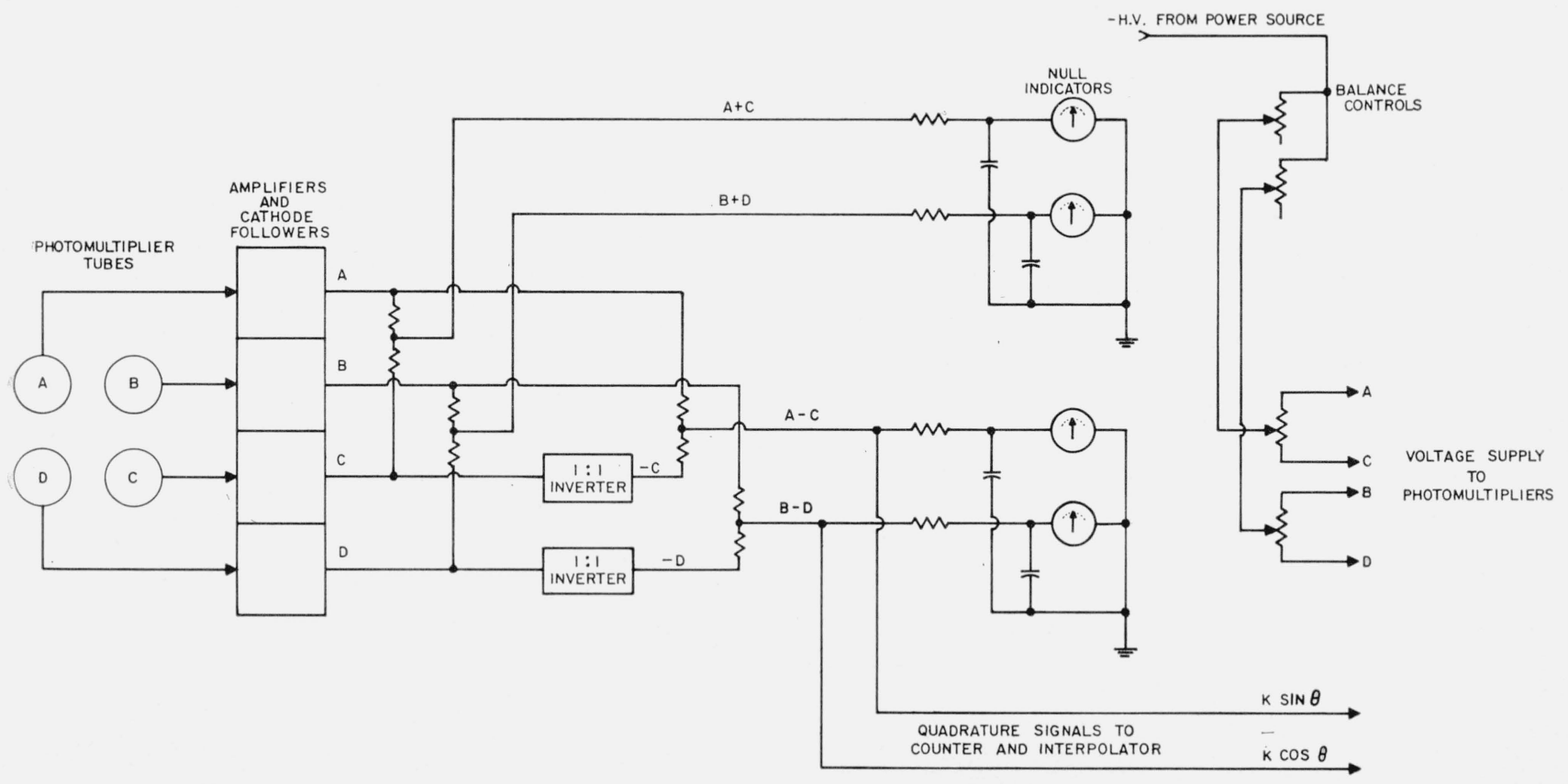

Figure 3. Block diagram of phototube and input circuits. 
able counter include a sufficiently high counting rate, convenient count indicators, and provision for automatically recording the count.

These requirements have been met in a counter using neon glow-transfer tubes. A counter was built for the experimental instrument because a suitable one was not commercially available. The counter has 6 reversible decade stages, allowing a count of $10^{6}$ without repeating. This exceeds the number of fringes in the range of the interferometer.

Counter Circuit Details. Figure 4 shows a block diagram of the counter. The sinusoidal phototube output signals are first transformed into rectangular waves by means of voltage sensitive trigger circuits. The pulses which operate the counter in the forward or reverse direction are generated by differentiating the rectangular waves and are distributed to the ADD or sUBTRACT counter inputs by the use of gating circuits. One of the fringe signals operates a pair of gates which allow transfer of pulses derived from the other fringe signal to the respective counter inputs depending on the polarity of the derivative pulse at the time the gates are open. As shown in figure 3 , the upper input becomes the gate signal and the lower one the count signal for the waveforms shown; the positive count pulse occurs during a positive gate signal. This common polarity operates the forward AND gate which in turn provides a pulse to the amplifiers for registering an ADD count at the glow-transfer tubes. The waveforms for reverse operation are such that the positive gate signal coincides with the negative count pulse. A polarity inverter reverses the negative pulse to positive and delivers it to the reverse AND gate. A pulse then goes to the amplifiers to register a SUBTRACT count.

The second stage shown in figure 4 (as well as all subsequent stages), possesses the reversibility feature. In order to provide these stages with the proper signals for reversible counting, 4 of the possible 10 -state positions from the previous counter stage are used as input signals. For counting in the forward direction, the sequence of the four states is $8,9,0,1$. Proceeding in a forward direction, the activation of state 8 at the first stage counter tube causes an output pulse to operate the lower flip flop into the position that renders the reverse AND gate

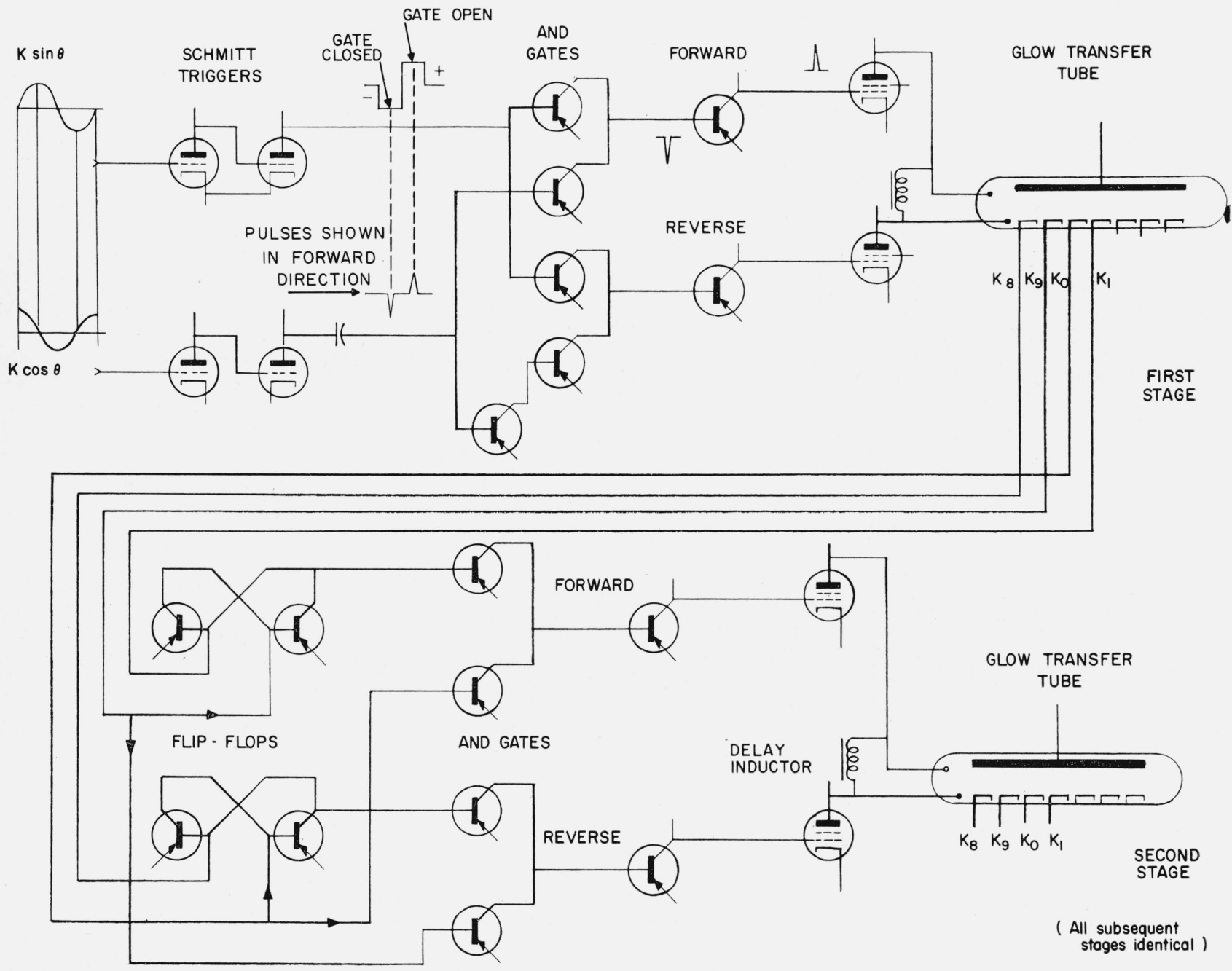

Figure 4. Block diagram of reversible counter. 
inoperative. On the 9th state the upper binary switch functions and places the forward AND gate in a receptive position. The 10 th or 0 state pulse passes through the forward AND gate and is registered at the counter tube in the manner previously described. The same 0-state pulse reverses the lower flip flop, placing the reverse gate in its receptive position. If the first counter tube should happen to reverse back to the 9 th state, a pulse from this state would pass through the reverse AND gate. The counter would reverse or subtract out one count. The complete arrangement is compatible to the extent that all counters can be made to oscillate in synchronism between 9 and 10 by the application of a single forward or reverse pulse at this unique combination of counter positions.

It may be worth while to say a few words about the glow-transfer counter tube. This electronic device has the ability to count input pulses and also visually indicate the totalized count. Its capacity is 1 decade, and it has provision for bringing out each of the 10 states for data transfer purposes and recording. In order for the tube to count, the input pulse is applied to 1 of 2 transfer electrodes. Connected across the transfer electrodes is an inductance-capacitance delay network whose function is to direct the same input pulse to the second transfer electrode, but with a time delay of several microseconds. It is the application of this time-staggered pair of pulses that causes the ionized-gas glow to be transferred from one state to the next. The transfer electrode requires a fast negative pulse with an amplitude of about $100 \mathrm{v}$. It is for this reason that a thermionic tube is used in the circuit in lieu of a transistor, with its limited voltage swing.

It was pointed out in the section on phototubes that, from a purely statistical viewpoint, a minimum value of design signal-to-noise ratio is established by the need to avoid miscounts. The probability of a noise pulse exceeding the fringe signal amplitude is one in $5 \times 10^{8}$ counts at the maximum rate for a signal-to-noise ratio of 6:1. With higher signal-tonoise ratios the probability of noise pulses exceeding the signal is negligible. It was further mentioned that the probability can be lowered by the use of a special trigger circuit. The relative noise immunity is achieved by a modification of the Schmitt ${ }^{3}$ trigger circuit increasing the hysteresis value. The hysteresis value is the voltage difference between the level at which the trigger circuit changes state in one direction and that for a change in the opposite direction. If the triggering levels are adjusted to be equally positive and negative from zero, and these are also at about half the peak voltage amplitude of the fringe signals, an optimum discrimination against noise results. In this case a rapidly repeated change of state which would be beyond the capability of the reversible counter can not occur unless a noise pulse exceeds the peak amplitude of the signal. If a small value of hysteresis were used, many rapid changes of state would occur with a small value of noise.

\footnotetext{
${ }^{3}$ Voltage sensitive trigger circuit.
}

\subsection{Interpolation}

If the precision of the fringe count is to be greater than $\pm \frac{1}{2}$ fringe, a means is required for determining the fractional fringe beyond the last integral count. In determining the count, some reference point on the sinusoidal function representing the fringe must be chosen. This is usually taken to be one of the points at which the function equals zero. In the past a number of methods of interpolation have been used, including a means of changing the effective path length of the interferometer until the fringe fiducial point is reached. The fraction is then read on a scale which is calibrated by changing the path by one whole fringe. In another method, sine and cosine voltages from the interferometer are displayed on a cathode ray tube, the two voltages being put on separate deflection axes. The display spot then follows a circle with the advance of the fringe, and the fringe fraction is equal to the polar angle measured around this circle, since there is one revolution per fringe. In order that the fraction may be accurately determined by this method, the voltages must accurately represent the sine and cosine values, the quadrature being accurate to a few degrees. Both of these methods are difficult to apply to automatic operation.

Since automatic data recording is a requirement for the application, development of an electronic means for determining the fringe angle was undertaken. The result is a relatively simple analog computer which indicates the angle in digital form to $1 / 100$ th fringe in 1 millisecond. The decimal fraction is indicated on counters of the same type as are used for the integral count, so that the method of data recording is the same for both.

The electronic operations in the conversion process may be described as taking place in three steps. Let $\phi$ represent the fringe angle; $\phi=360^{\circ}$ for a displacement of one fringe. First, the two input voltages $A \sin \phi$ and $A \cos \phi$ from the phototube circuits are used to determine the amplitude of quadrature components of an alternating voltage with an arbitrary angular frequency $\omega_{1}$. The result is $A \sin \phi \sin \omega_{1} t$ and $A \cos \phi \cos \omega_{1} t$. Second, the difference between these voltages is taken. The result can be expressed by a trigonometrical identity:

$$
A \sin \phi \sin \omega_{1} t-A \cos \phi \cos \omega_{1} t \equiv A \cos \left(\omega_{1} t+\phi\right) .
$$

The third step is to measure the phase shift between this sum and $\sin \omega_{1} t$, since the phase difference is equal to the fringe angle $\phi$.

Interpolator Circuits. A block diagram of the interpolator is shown in figure 5 .

Electronic balanced modulation or multiplication is generally difficult and subject to errors in linearity. A technique which is believed to be new greatly simplifies the generation of the products required in the first step. The method makes use of linear gates in the resonance paths of LC networks. The principle of generation is shown in figure 6 .

Two resonant LC circuits, one for each of the quadrature signals, are excited to oscillation simul- 


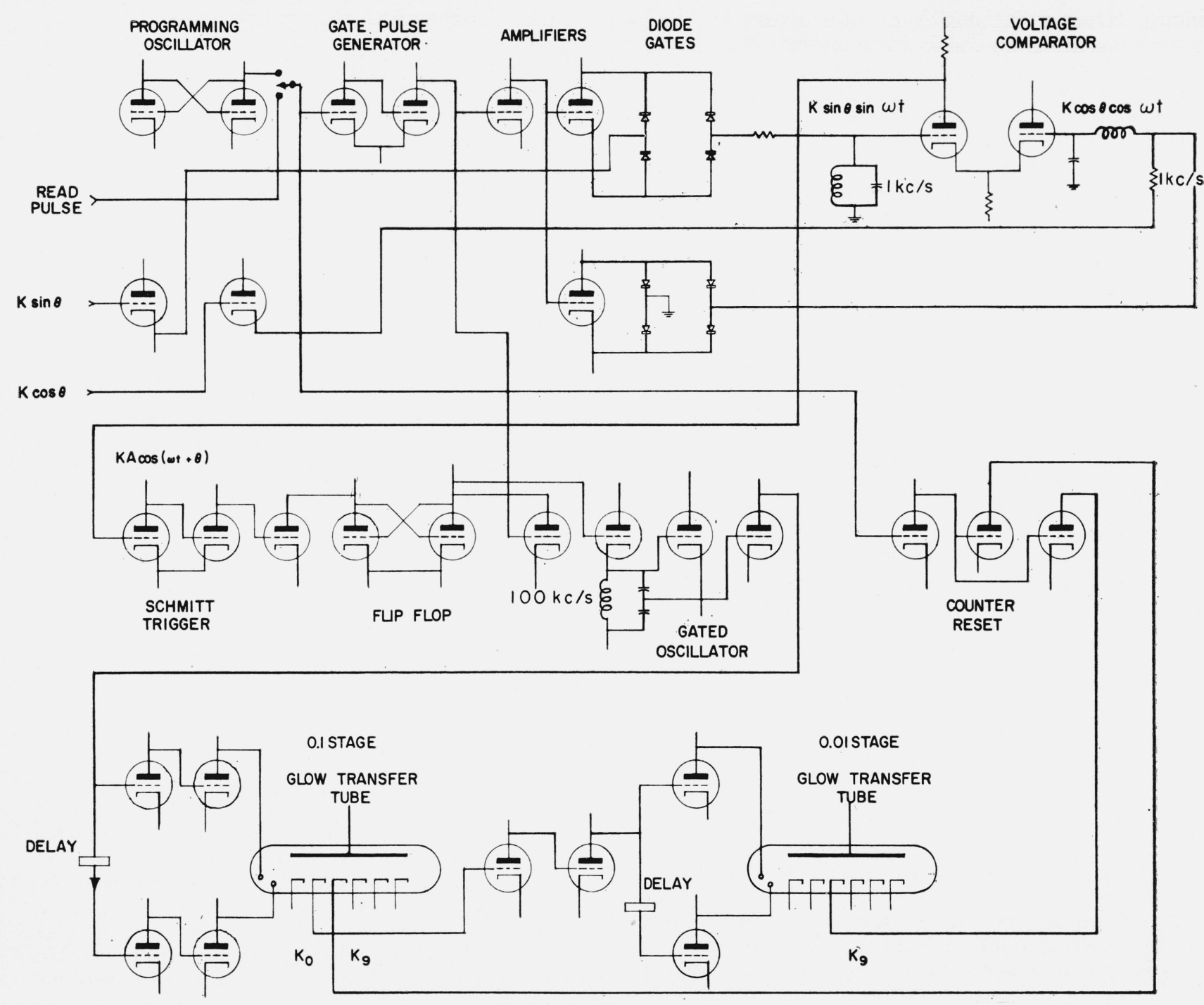

Frgure 5. Block diagram of digital interpolator.



Figure 6a. Generator for $A \sin \theta \sin \omega_{1} t$.

taneously, the amplitudes being proportional to the values of $\sin \phi$ and $\cos \phi$. The arrangement of gating is such that one LC circuit generates a cosine wave at angular frequency $\omega_{1}$ and the other a sine wave at the same frequency. The energy by which the resonant circuits are excited is introduced by a current proportional to $\sin \phi$ in the inductor of one, and a voltage proportional to $\cos \phi$ on the capacitor

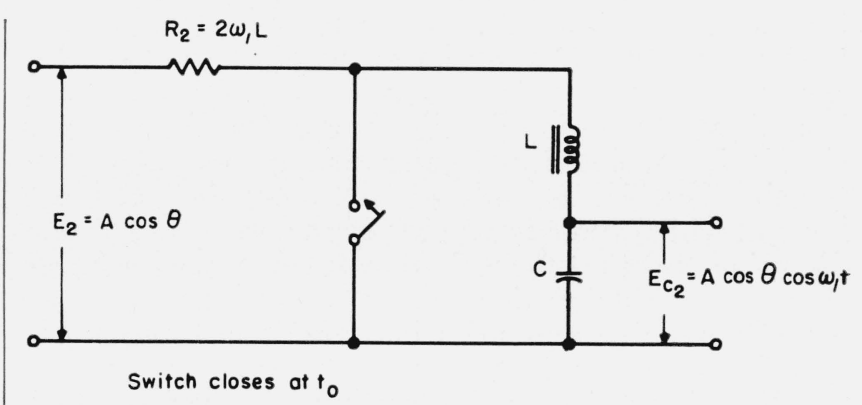

Figure 6b. Generator for $A \cos \theta \cos \omega_{1} t$.

of the other. At a time $t_{0}$, an initiating pulse is generated either manually or automatically, causing operation of the gating circuits and allowing the LC circuits to oscillate at their resonance frequency for a period of at least 1 cycle. The voltage on the respective capacitors then becomes $\sin \phi \sin \omega_{1} t$ and $\cos \phi \cos \omega_{1} t$. The difference between these is com- 
pared with zero by means of a voltage sensitive trigger circuit, and the time interval to the first positive zero crossing measured. The time intervals measured by the use of an alternating voltage of angular frequency $100 \omega_{1}$ and 2 decade cycle counters which indicate the fringe angle in decimal form.

Operation of the interpolator is not necessary during the process of counting, but is needed only to determine the fractional fringe at either end of a measurement. One initiating pulse at each position is sufficient to read the fraction, although it is convenient to apply repeated pulses at perhaps 15 or $20 / \mathrm{sec}$ when watching the count visually. The appearance is that of a continuous reversible fringe count reading to 0.01 fringe.

\subsection{Ambiguity in the Fringe Count}

Since there is hysteresis in the trigger circuits of the integral counter, the purpose of which is to eliminate multiple triggering at zero crossings of the fringe signals, the indicated count does not change exactly at the fringe fiducial point used as a reference for the interpolator. This results in an ambiguity of a unit count in the region near the zero point of each fringe.

In order to insure that the same fringe zero reference point is used for both the integral counter and interpolator, it is necessary to make use of information developed by the interpolator. In forward counting, if the interpolator indicates that the zero point is passed, the integral counter must be forced to indicate the next higher count. In reverse counting, the reverse must be true when the interpolator indicates less than zero. This is necessary only when an interpolation is made, since only the final count is recorded.

The antiambiguity circuit consists of a set of pulse generators responding to the $0.8,0.9,0.0$, and 0.1 states of the second glow-transfer tube of the interpolator counter. At the end of the processing time of the interpolator, a pulse is generated which is negative if the interpolation indicates 0.8 or 0.9 , and positive if the indication is 0.0 or 0.1 One such pulse is applied to the counter trigger circuit and superimposed on the fringe signal. The pulse forces the signal voltage to exceed the triggering threshold in the direction of the count, eliminating the ambiguity.

\subsection{Interpolation Bandwidth}

The signal-to-noise ratio allowable in the input of the interpolator depends on the desired accuracy of interpolation. The value of the sine or cosine input voltage may not vary by more than \pm 6 percent of the maximum amplitude if the reading is to be accurate to $\pm 1 / 100$ th fringe. Allowing for the statistical character of the noise and the characteristics of the counter and interpolator, a ratio of approximately 1000:1 is required between the bandwidth for counting reliably and the bandwidth for interpolating to $1 / 100$ th fringe. The maximum fringe rate when counting or interpolating has this same ratio.

\subsection{Parallelism}

Measurement of length with a Michelson interferometer is accomplished by moving one of the plane mirrors which is mounted on a carriage, along a set of precise ways. Angular errors of motion of the carriage affect the geometry of the interferometer, and these may amount to as much as a micron per centimeter. If the error is greater than one-half wavelength across the mirror, the fringes are entirely lost and counting is impossible.

Cube corners used as reflectors maintain the fringe pattern in spite of angular errors, but for the application it was considered more desirable to maintain parallelism by correcting the error. If this is done, plane mirrors can be used. Also it is made certain that the position of a reference point on the moving carriage on which the mirror is mounted always maintains a fixed relationship to the effective plane of reflection so that length measurements may be made accurately.

In the instrument described here the angular errors are eliminated by means of a servomechanism which includes first, a means for detecting the error, and second, a set of actuators to provide the necessary correction. A voltage indicating the error is obtained from a comparison of the phase of the fringes detected at diagonally opposite corners of the mirror. For this purpose the interferometer beam is divided into 4 quadrants with a separate phototube for each, thus dividing the instrument into essentially 4 separate interferometers working simultaneously. If the mirror is in perfect alinement, there is no phase error detected, but if there is an angular error and one mirror quadrant leads its diagonally opposite quadrant, a phase difference occurs between the respective phototube signals. An error of $1 / 100$ th fringe is easily detected.

The actuators alter the relationship between the mirror and the carriage by an amount nearly equal to the angular error of the carriage so that the mirror does not depart from parallelism by more than is necessary to produce an error signal. Barium titanate is used for the actuators in the experimental equipment, but is limited to a few microns in its range of motion. Small hydraulic acutators have also been used successfully for this purpose. In addition to their simplicity, both types of actuators have an advantage in that there is a minimum of energy dissipated in their operation, and none is required to hold a fixed position.

Since in this equipment the phase is determined from the relative timing of the axis crossings of the phototube signals, the mirror must be moving at the time of correction. It is necessary, therefore, to incorporate some means of maintaining the correction voltage or pressure if the parallelism is to be held for long periods at rest. A suitable voltage memory is a motor-driven potentiometer controlled by the error signal. In the case of the hydraulic actuators, flexible tubes containing a fluid are raised or lowered to provide the required pressure. 
Circuit Description. In figure 7, $A$ and $C$ represent one diagonal pair of phototube outputs, and $B$ and $D$ the other. The discussion for one pair will hold equally well for the other. Each of the amplified phototube outputs is applied to a voltage sensitive trigger tube in order to generate square waves, the transitions occurring very close to the zero crossings of the sinusoidal fringe signal. The networks following the amplifiers form the sum of the square waves, $A^{\prime}$ and $C^{\prime \prime}$, to their opposite derivatives, $d C^{\prime} d t$ and $d A^{\prime} d t$. In figure 7 , the waveforms shown are for the condition in which the mirrors are out of parallelism in a direction such that signal $C$ lags signal $A$ by slightly more than the normal $180 \mathrm{deg}$. Note that for this condition, the $C^{\prime}+\left(d A^{\prime} d t\right)$ output has a sharp positive pulse on top of the pedestal. If the mirror were to be tilted out of parallelism in a direction opposite to the previously assumed direction, the upper waveform, $A^{\prime}+\left(d \dot{C}^{\prime} d t\right)$, would have the positive pulse on its pedestal.

For a mirror travel restricted to one direction only, the circuits described would be sufficient to provide a signal for correction purposes. However, for bidirectional travel, the correction sense must be reversed if the direction is reversed. This is done by means of a circuit in which gates are actuated by signals from the $B$ and $D$ signal channels. As shown in figure 7 , the gate outputs are combined by diodes to form two separate output lines. Signals appearing at each line are further amplified (in addition to a polarity inverter in one line), and are recombined at the grid of a cathode follower. The positive or negative output of the latter (depending upon mirror tilt and travel direction) is used as the error control signal in the servo loop for maintaining the mirror position.

As mentioned previously, the servo loop includes a motor-driven voltage control for the purpose of providing position memory of mirror parallelism while the interferometer is at rest. The $\mathrm{d}-\mathrm{c}$ output of the control in turn is amplified to a potential of several hundred volts in order to drive the barium titanate transducer.

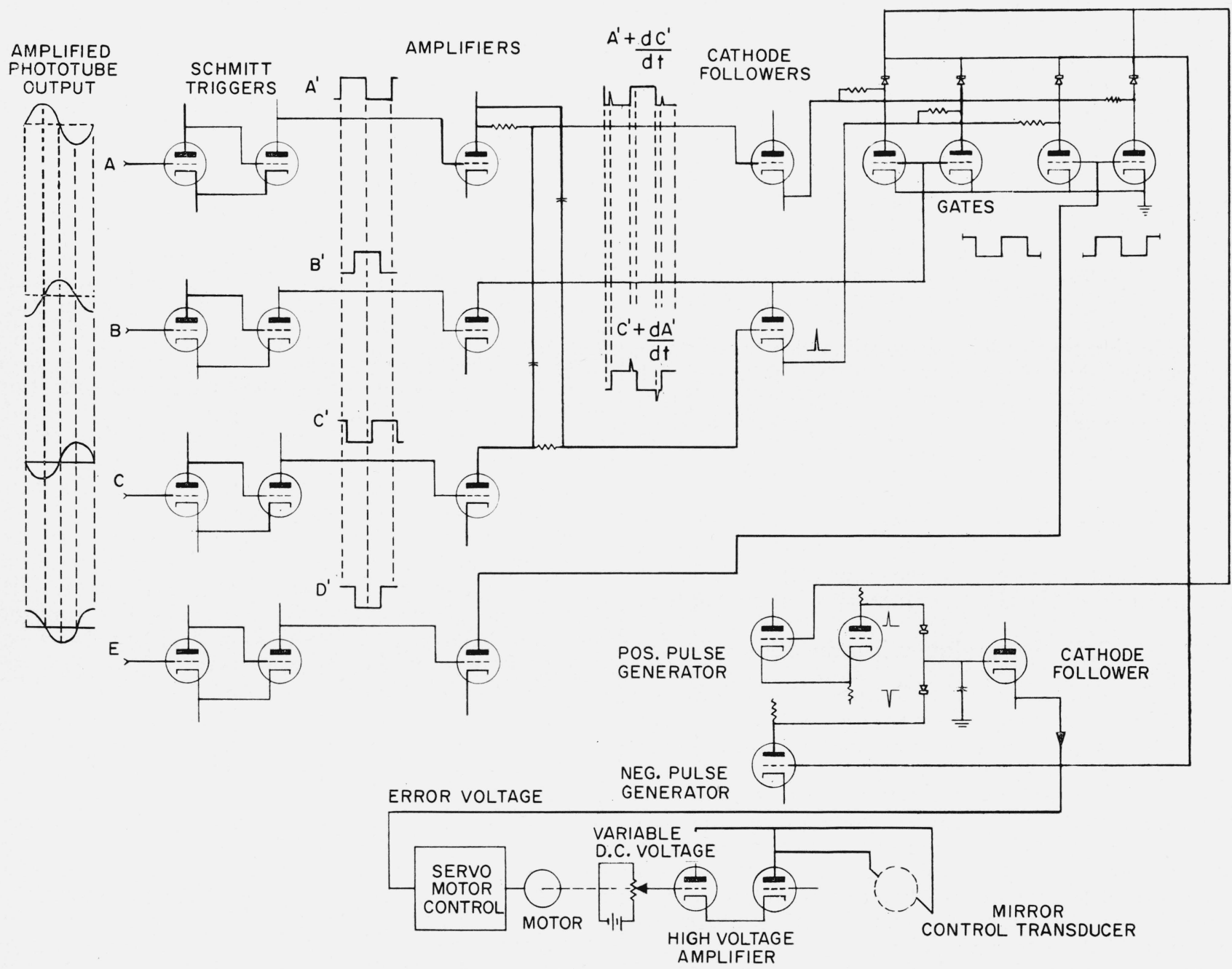

Figure 7. Mirror parallelism control circuit. 
The phase detector circuit is capable of resolving a phase difference of 0.01 fringe. With a mirror $3-\mathrm{cm}$ square and with green light, this is equivalent to $10^{-7}$ radians, or about $0.02 \mathrm{sec}$ of are.

\section{Accuracy of Measurements}

Since a length measured by the interferometer is a comparison with respect to the wavelength of the light source, any variation in the wavelength in the interferometer affects the accuracy of measurement. The stability of the wavelength of the krypton lamp may be 1 part in $10^{9}$, whereas the mercury 198 lamp is affected by the presence of a carrier gas by a few parts in $10^{7}$. The $\mathrm{Hg} 198$ lamp is calibrated, however, and may be relied on to the extent that the argon pressure is known. The wavelength is given to one part in $10^{8}$.

A greater variation in the wavelength in the interferometer is due to the refractive index of the ambient air. The refractive index error is proportional to the density of the medium and is therefore affected by the temperature, the pressure, and the proportion of other constituents such as $\mathrm{CO}_{2}$ and water vapor. If the wavelength is to be known to one part in $10^{8}$, the atmospheric pressure must be known to approximately $0.0025 \mathrm{~cm}$ of $\mathrm{Hg}$, the air temperature to $0.01{ }^{\circ} \mathrm{C}$, water vapor content to $0.25 \mathrm{~mm}$ partial pressure, and $\mathrm{CO}_{2}$ to $1 / 100{ }^{4}$

\section{Aperture Error Correction}

Another factor influencing the accuracy of measurement is characteristic of the interferometer. In an interferometer in which a phototube is used to detect the fringes, the number of fringes counted is not exactly equal to the distance of mirror travel divided by one-half the wavelength of light, since the phototube receives light from more than the central fringe. The ratio of the count obtained to the true count is a constant factor which depends on the degree of collimation of the light in the interferometer. The difference is significant since the numerical value of the necessary correction is generally greater than one part in $10^{6}$ and may total more than one fringe in a given measurement. A correction factor for the error can be applied which is fortunately a constant for a given angular aperture and applies equally throughout the range of measurement. It is also the same for a fixed aperture for any wavelength of light.

If the source were a point on the axis of the collimator, the fringes would truly represent the separation of the interferometer plates, but since the brightness of the source is finite, the light flux obtained from a point on the axis would be zero. In order that the light flux through the interferometer be finite, the source must be broad, and as a result, essentially all of the rays that are used are nonparallel to the axis. The average path of rays that are seen by the phototube is thus at an angle to the axis and the average optical path difference is not equal to that normal to the axis.

\footnotetext{
${ }^{4}$ W. F. Meggers, C. G. Peters, Bull. BS 14, 697 (1918); B. Edelen, J. Opt. Soc. Am, 43, 339 (1953).
}

Integration of the light flux received by a phototube in an interferometer has been described in a number of published papers. ${ }^{5}$ An expression for the fringe contrast versus optical path difference and aperture is derived in appendix 2. The magnitude of the length correction is given.

\section{Conclusion}

The interferometer described here is applicable to uses in which the fringe count and interpolation is required to be continuously available, as in systems where fully automated control is desired.

Further improvement in the measuring ability of the instrument will be in the direction of extended range and in the speed of counting. Such improvement depends primarily on the light source, the important factors being the monochromaticity of the radiation and the flux per Angstrom of spectrum per unit solid angle from a source of unit area. Both of these factors are characteristic of the coherent sources now being developed elsewhere and which should be available in the not too distant future.

With present light sources, there is an approximately reciprocal relationship between the maximum range of fringe counting and the speed of counting, the acceptance angle of the collimator aperture being the variable parameter. This is true within the limitations of (1), the length over which interference can be obtained, and (2), the maximum rate of the counter.

In the application of the interferometer to scale calibration, it is used in conjunction with a photoelectric microscope which has comparable precision. Measurement of a scale which is longer than the maximum range of the interferometer is done by measuring sections of the scale, allowing the measurements to overlap with several lines in common. The error in adding the sections to derive the total is thereby minimized by the redundancy in reading the lines.

\section{Appendix 1. Relation of Phototube Signals}

If the source intensity is $I$ and the fringe contrast $m$, the currents in the phototubes $A, B, C, D$ are respectively:

$$
\begin{aligned}
& k_{A} I\left(1+m_{A} \sin \phi\right) \\
& k_{B} I\left(1+m_{B} \cos \phi\right) \\
& k_{C} I\left(1-m_{C} \sin \phi\right) \\
& k_{D} I\left(1-m_{D} \cos \phi\right)
\end{aligned}
$$

When balance adjustments are made, $k_{A}, k_{B}, k_{C}, k_{D}$ become equal.

The difference between $A$ and $C$ is then:

$$
A-C=k I\left(m_{A}+m_{C}\right) \sin \phi
$$

5 M. Francon, Rev. D’Opt. 26, 434 (1947). E. R. Peck, S. W. Obetz, J. Opt. Soc. Am. 43, 505 (1953). Joseph H. Jaffe, J. Opt. Soc. Am. 43, 1170 (1953). R. D. Soc. Am. 43, 505 (1953). Joseph H. Jaffe, J. Opt. Soc. Am. 43, 1170 (1953). R. D.
Huntoon, A. Weiss, J. Opt. Soc. Am. 44, 4 (1954). G. R. Harrison, G. W. Stroke. Huntoon, A. Weiss, J. Opt. Soc. Am. 44, 4 (1954), G. R. Harrison, G. W. Stroke.
J. Opt. Soc. Am. 45, 112 (1955). E. R. Peck, J. Opt. Soc. Am. 45, 931 (1955).

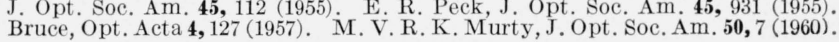


Also

$$
B-D=k I\left(m_{B}+m_{D}\right) \cos \phi
$$

The result is a sine function and a cosine function of $\phi$ with baselines equal to zero and amplitudes proportional to $I$.

\section{Appendix 2. Calculation of the Correction Factor}

A ray from a point within the aperture leaving the collimating lens at an angle $\theta$ with respect to the axis of the system passes through the interferometer at this same angle. Since the optical path difference in the interferometer varies as the cosine of this angle, the average optical path difference for the complete set of rays is less than that for a ray which is parallel to the axis. The total light flux reaching the phototube through the aperture and interferometer is found by integrating the interference function over the angular aperture.

The point intensity of a ray parallel to the axis is proportional to

$$
1+m \cos \frac{2 \pi D}{\lambda}
$$

where $D$ is twice the mirror separation, $\lambda$ is the wavelength, and $m$ is a factor representing the fringe contrast for a perfectly collimated beam at an optical path difference equal to $D$. For any ray the expression becomes:

$$
1+m \cos \left(\frac{2 \pi D}{\lambda} \cos \theta\right)
$$

Integrating over a sphere from zero to $\theta_{1}$, the limit of the angular aperture,

$$
\begin{aligned}
\text { Flux } & =\int_{\theta}^{\theta_{1}}\left[1+m \cos \left(\frac{2 \pi D}{\lambda} \cos \theta\right)\right] \sin \theta d \theta \\
& =1-\cos \theta_{1}+m \frac{\sin \frac{2 \pi D}{\lambda}-\sin \left(\frac{2 \pi D}{\lambda} \cos \theta_{1}\right)}{\frac{2 \pi D}{\lambda}}
\end{aligned}
$$

By trigonometric identity this is equal to

$$
1-\cos \theta_{1}+2 m \frac{\sin \left(\frac{2 \pi D}{\lambda} \cdot \frac{1-\cos \theta_{1}}{2}\right)}{\frac{2 \pi D}{\lambda}} \cos \left(\frac{2 \pi D}{\lambda} \cdot \frac{1+\cos \theta_{1}}{2}\right)
$$

This is a cosine function of $D$ the optical path difference in which the argument is similar to that of the intensity function for an axial ray except for the factor $\left(1+\cos \theta_{1}\right) / 2$. This is the correction factor by which the fringe count must be multiplied in order to obtain a true measurement of length in half wavelengths of light. It is approximately equal to

$$
1-\frac{\theta_{1}^{2}}{4}+\frac{\theta_{1}^{4}}{48}
$$

The expression also shows the variation of the amplitude of the cosine function with respect to $D$, assuming no reduction in contrast for other reasons. The amplitude becomes zero when $D=\lambda /\left(1-\cos \theta_{1}\right) \cong$ $2 \lambda / \theta_{1}^{2}$. The maximum useful counting distance $D_{\max }$ is considerably less than this, perhaps $\lambda / \theta_{1}{ }^{2}$ where the amplitude is about half the maximum.

\section{Bibliography}

1951. Wood, R. N., A photoelectric recording interferometer of measurement of dimensional changes, NBS J. Research 4\%, 2.

1951. Harrison, G. R., J. E. Archer, Interferometric calibration of precision screws and control of ruling engines, J. Opt. Soc. Am. 41, 8.

1952. Root, Elihu III, Measuring device employing light interference fringes, U.S. Patent No. 2,604,004.

1953. Peck, E. R., S. Wendell Obetz, Wavelength or length measurement by reversible fringe counting, J. Opt. Soc. Am. 43, 6.

1953. Branin, F. H. Jr., A bidirectional electronic counter for use in optical interferometry, J. Opt. Soc. Am. 43, 10.

1954. Huntoon, R. D., A. Weiss, J. Wray Smith, Electronic fringe interpolator for an optical interferometer, J. Opt. Soc. Am. 44, 4.

1957. Harrison, G. R., et al., Ruling of large diffraction gratings with interferometric control, J. Opt. Soc. Am. 47, 1.

1958. Eisner, R. L., Reversible photoelectric fringe counter, Rev. of Sci. Inst. $\mathbf{2 9}, 6$.

1959. Hara, Ko, D. S. Smith, Length measurement by fringe counting, Rev. Sci. Inst. 30, 8.

1959. DuMont Multiplier Photo Tubes, Allen B. DuMont Laboratories, Inc., Clinton, N. J. 